\title{
Entanglement and Quantum Discord of Two Moving Atoms
}

\author{
Nour Zidan \\ Mathematics Department, Faculty of Science, Sohag University, Sohag, Egypt \\ Email: nazidan@yahoo.com
}

Received 27 June 2014; revised 31 July 2014; accepted 12 August 2014

Copyright (C) 2014 by author and Scientific Research Publishing Inc.

This work is licensed under the Creative Commons Attribution International License (CC BY). http://creativecommons.org/licenses/by/4.0/

(c) (7) Open Access

\begin{abstract}
In this paper we discuss two measures for the quantumness of correlations (quantum discord and entanglement) of two isolated moving atoms which are initially in Werner state. We compare and analyze the effect of the atomic motion in quantum discord and entanglement. The results show that the atomic motion related to the field mode structure parameter can make the entanglement and quantum discord evolve periodically. The results also indicate that the entanglement suddenly disappears during the evaluation but quantum discord remains non zero, so quantum discord is considered as indicator of disentanglement.
\end{abstract}

Keywords

Quantum Discord, Concurrence, Moving Atoms, Werner State

\section{Introduction}

Entanglement is a kind of quantum correlation that has been regarded as a valuable resource for quantum information processing [1]. However, it is was shown both theoretically [2] and experimentally [3] that some mixed separable states allow one to realize the advantages of quantum algorithms in comparison with their classical analogies and quantum nonlocality has been observed in the systems without entanglement [4] [5]. Such observations suggest us to conclude that the entanglement is not the only type of quantum correlation for quantum technology. A new but promising candidate is quantum discord first introduced by Ollivir and Zurek, which is used to quantify all nonclassical correlation in a system [6]. Quantum discord is built on the fact that two classical equivalent ways of defining the mutual information turn out to be inequivalent in the quantum domain. It is observed that quantum discord is a more general measure of quantum correlation that may include entanglement but is an independent one. Moreover, quantum discord provides a larger region of quantum states with nonclassical correlations, and a nonzero quantum discord but not entanglement may be responsible for the efficiency of 
a quantum computer [3]-[7]. Therefore, quantum discord could be a new resource for quantum information processing. Recently, numerous works have been devoted to the study of quantum discord [8]-[12]. Usually, quantum discord is somewhat difficult to calculate and we cannot obtain analytical solutions. But in [13], the authors evaluated the quantum discord for a family of two-qubit states and obtained analytical formulas. For more general quantum states, such as the so-called $X$ states, the authors [14]-[21] derived explicit expressions for quantum discord and found that the quantum discord, entanglement and classic correlation were independent measures of correlations with no simple relative ordering. An extreme example is realized in the search of maximally discordant mixed states. It has revealed that states maximizing the quantum discord for given value of the classical correlations do not maximize the entanglement and are, in some cases, even separable [22]. Very recently a reliable and effective algorithm for the evaluation of the quantum discord of the general two-qubit states was presented, where the optimization problem for the quantum discord was expressed in a compact form [23]. In this work, we calculate and compare the time evolution of quantum discord and concurrence of two moving isolated atoms. This paper is organized as follows. In Section 2, we give the model and its solution. In Section 3, we give two types of measures including quantum discord and concurrence to analyze quantum correlation. Numerical results and discussion are given in Section 4. Finally we conclude in Section 5.

\section{The Model and Its Solution}

We consider two identical moving two-level atoms labeled $A$ and $B$ interact with two single-mode thermal cavity fields labeled $a$ and $b$. Each atom-cavity system is isolated (atom $A$ at a constant velocity interacting only with field $a$ and similarly for the atom $B$ and the field $b$ ). For simplicity, we suppose that these two atoms be coupled to the field with the same coupling constant $g$, and the field mode be resonant with the transition frequency of both atoms. The interaction Hamiltonian under the rotating wave approximation $(\hbar=1)$ is

$$
H_{j}^{\text {Int }}=g f_{j}(z)=\left(a_{j}^{\dagger} \sigma_{j}^{-}+\sigma_{j}^{+} a_{j}\right), \quad(j=A, B)
$$

where $a_{j}^{\dagger}\left(a_{j}\right)$ is the creation (annihilation) for $j^{\text {th }}$ mode of the cavity field and $\sigma_{j}^{+}, \sigma_{j}^{-}$are the raising and lowering operators respectively. $f_{j}(z)$ denotes the mode structure of cavity field which can be defined specially as [24]

$$
f_{j}(z)=f_{j}(v t)=\sin \left(\frac{p_{j} v_{j} \pi t}{L_{j}}\right),
$$

where, $v_{j}$ denotes the velocity of the $j^{\text {th }}$ atom and $p_{j}$ represents the half-wave number of cavity field with length $L_{j}$. We restrict our study for atomic in the $z$-axis direction, so that only the $z$-dependent field mode function needs to be taken into account. For simplicity we assume that $v_{A}=v_{B}=v, L_{A}=L_{B}=L$ and $p_{A}=p_{B}=p$. Using standard technique, it can show that $H_{j}^{\text {Int }}$ in Equation (1) gives rise to the following time evolution operator

$$
U_{j}(t)=\exp \left[-i \int_{0}^{t} H_{j}^{I n t} \mathrm{~d} t^{\prime}\right]=\left(\begin{array}{cc}
\cos \left[g \theta(t) \sqrt{a_{j} a_{j}^{\dagger}}\right] & \frac{-i \sin \left[g \theta(t) \sqrt{a_{j} a_{j}^{\dagger}}\right] a_{j}}{\sqrt{a_{j} a_{j}^{\dagger}}} \\
\frac{-i \sin \left[g \theta(t) \sqrt{a_{j}^{\dagger} a_{j}}\right] a_{j}^{\dagger}}{\sqrt{a_{j}^{\dagger} a_{j}}} & \cos \left[g \theta(t) \sqrt{a_{j}^{\dagger} a_{j}}\right]
\end{array}\right),
$$

where, $\theta(t)=\int_{0}^{t} f\left(v t^{\prime}\right) \mathrm{d} t^{\prime}=\frac{i}{p}[1-\cos (\beta t)]$ and $\beta=p \pi v / L$. For a particular choice of the atomic motion velocity $v=g L / \pi, \theta(t)$ becomes

$$
\theta(t)=\frac{1}{p g}[1-\cos (p g t)]
$$

We assume that the two atoms are initially in the Werner state 


$$
\boldsymbol{\rho}_{A B}(0)=\boldsymbol{\rho}_{W}(0)=r\left|\psi^{+}\right\rangle\left\langle\psi^{+}\right|+\frac{1-r}{4} \boldsymbol{I}_{4} .
$$

Here, $\left|\psi^{+}\right\rangle=(|10\rangle+|01\rangle) / \sqrt{2}, \quad \boldsymbol{I}_{4}$ is the $4 \times 4$ identity matrix and $r$ varies from 0 to 1 , and for $r=0$ the Werner becomes maximally states, while for $r=1$ they are the well known Bell states. The two cavity fields $a$ and $b$ are initially in single-mode thermal field states

$$
\boldsymbol{\rho}_{a}(0)=\sum_{n} P_{n}|n\rangle\left\langle n\left|, \quad \boldsymbol{\rho}_{b}(0)=\sum_{m} P_{m}\right| m\right\rangle\langle m| .
$$

The weight function $P_{n}$ and $P_{m}$ are,

$$
P_{n}=\frac{\bar{n}^{n}}{(1+\bar{n})^{n+1}}, \quad P_{m}=\frac{\bar{m}^{m}}{(1+\bar{m})^{m+1}},
$$

where $\bar{n}=\left[\exp \left(\hbar \omega_{f} k_{\beta} T_{a}\right)-1\right]^{-1}$ and $\bar{m}=\left[\exp \left(\hbar \omega_{f} k_{\beta} T_{b}\right)-1\right]^{-1}$ are the mean photon numbers of the two thermal cavity fields $a$ and $b$ corresponding to the equilibrium cavity temperatures $T_{a}$ and $T_{b}$ respectively, $k_{\beta}$ is the Boltzmann constant and $\omega_{f}$ is the frequency of the field.

Using the evolution operator, we can generate the density operator of the system at time $t$ as

$$
\boldsymbol{\rho}(t)=U_{A}(t) \otimes U_{B}(t)\left[\boldsymbol{\rho}_{A B}(0) \otimes \boldsymbol{\rho}_{a}(0) \otimes \boldsymbol{\rho}_{b}(0)\right] U_{B}^{\dagger}(t) U_{A}^{\dagger}(t) .
$$

We can obtain the reduced density matrix for the two atoms at time $t$ by tracing over the field variables. Under the standard basis $\{|11\rangle,|10\rangle,|01\rangle,|00\rangle\}$ the reduced density matrix $\boldsymbol{\rho}_{A B}(t)$ can be obtained in the form

$$
\boldsymbol{\rho}_{A B}(t)=\operatorname{tr}_{f} \boldsymbol{\rho}(t)=\left(\begin{array}{cccc}
\rho_{11} & 0 & 0 & 0 \\
0 & \rho_{22} & \rho_{23} & 0 \\
0 & \rho_{32} & \rho_{33} & 0 \\
0 & 0 & 0 & \rho_{44}
\end{array}\right),
$$

where the matrix elements are expressed as follow

$$
\begin{aligned}
& \rho_{11}=\frac{1-r}{4} \sum_{n} \sum_{m}\left(P_{n} P_{m} C_{n+1}^{2} C_{m+1}^{2}+P_{n-1} P_{m-1} S_{n-1}^{2} S_{m-1}^{2}\right)+\frac{1+r}{4} \sum_{n} \sum_{m}\left(P_{n} P_{m-1} C_{n+1}^{2} C_{m-1}^{2}+P_{n-1} P_{m} S_{n-1}^{2} S_{m+1}^{2}\right), \\
& \rho_{22}=\frac{1-r}{4} \sum_{n} \sum_{m}\left(P_{n} P_{m} C_{n+1}^{2} S_{m+1}^{2}+P_{n-1} P_{m} S_{n-1}^{2} C_{m-1}^{2}\right)+\frac{1+r}{4} \sum_{n} \sum_{m}\left(P_{n} P_{m} C_{n+1}^{2} C_{m}^{2}+P_{n-1} P_{m} S_{n-1}^{2} S_{m+1}^{2}\right), \\
& \rho_{33}=\frac{1-r}{4} \sum_{n} \sum_{m}\left(P_{n} P_{m} S_{n+1}^{2} C_{m+1}^{2}+P_{n} P_{m-1} C_{n}^{2} S_{m-1}^{2}\right)+\frac{1+r}{4} \sum_{n} \sum_{m}\left(P_{n} P_{m-1} S_{n+1}^{2} S_{m-1}^{2}+P_{n} P_{m} C_{n}^{2} C_{m+1}^{2}\right), \\
& \rho_{44}=\frac{1-r}{4} \sum_{n} \sum_{m}\left(P_{n} P_{m} S_{n+1}^{2} S_{m+1}^{2}+P_{n} P_{m} C_{n}^{2} S_{m}^{2}\right)+\frac{1+r}{4} \sum_{n} \sum_{m}\left(P_{n} P_{m} S_{n+1}^{2} C_{m}^{2}+P_{n} P_{m} C_{n}^{2} S_{m+1}^{2}\right), \\
& \rho_{23}=\frac{r}{2} \sum_{n} \sum_{m}\left(P_{n} P_{m} C_{n} C_{m} C_{n+1} C_{m+1}\right),
\end{aligned}
$$

with $\rho_{32}=\rho_{23}^{*}$ (the asterisk denotes complex conjugation) and all matrix elements are zero, $P_{n}$ and $P_{m}$ are given in Equation (7), and the time-dependent operations are expressed by

$$
\begin{array}{ll}
C_{n}=\cos [\sqrt{n} g \theta(t)], & S_{n}=\sin [\sqrt{n} g \theta(t)] \\
C_{m}=\cos [\sqrt{m} g \theta(t)], & S_{m}=\sin [\sqrt{m} g \theta(t)],
\end{array}
$$

where $\theta(t)$ is given in Equation (4).

\section{Quantum Discord and Entanglement}

In the following, we will investigate the quantum discord and entanglement between two moving atoms by using 
above results. In order to follow the entanglement of the two atoms, we choose the Wootters entanglement measure [25], the concurrence $C$ defined as

$$
C=\max \left(0, \lambda_{1}-\lambda_{2}-\lambda_{3}-\lambda_{4}\right),
$$

where the quantities $\lambda_{1} \geq \lambda_{2} \geq \lambda_{3} \geq \lambda_{4}$ are the square roots of the eigenvalues of the matrix $\boldsymbol{R}=\boldsymbol{\rho}_{A B}\left(\sigma_{y} \otimes \sigma_{y}\right) \sigma_{A B}^{*}\left(\sigma_{y} \otimes \sigma_{y}\right)$. The concurrence $C=0$ corresponds to an unentangled states and $C=1$ to a maximally entangled state.

On the other hand, quantum discord is based on the difference between the quantum mutual information and the classical correlation [26]. For a two-qubit quantum system, the total correlation is measured by their quantum mutual information

$$
L\left(\boldsymbol{\rho}_{A B}\right)=S\left(\boldsymbol{\rho}_{A}\right)+S\left(\boldsymbol{\rho}_{B}\right)-S\left(\boldsymbol{\rho}_{A B}\right),
$$

where $\boldsymbol{\rho}_{A(B)}$ and $\boldsymbol{\rho}_{A B}$ denote the reduced density matrix of $A(B)$ and the density of the bipartite system respectively, and $S(\rho)=-\operatorname{tr}\left(\rho \log _{2} \rho\right)$ is the von Neumann entropy. Quantum discord, which quantifies the quantumness of correlation between $A$ and $B$, is then defined as the difference between the total correlation and classical correlation,

$$
Q D\left(\boldsymbol{\rho}_{A B}\right)=L\left(\boldsymbol{\rho}_{A B}\right)-C C\left(\boldsymbol{\rho}_{A B}\right) .
$$

Here the classical correlation $C C\left(\boldsymbol{\rho}_{A B}\right)$, which quantifies the maximum information that one can obtain from the composite system by measurement on one of the subsystem, can be expressed as

$$
C C\left(\boldsymbol{\rho}_{A B}\right)=\max _{\left\{B_{k}\right\}}\left[S\left(\boldsymbol{\rho}_{A}\right)-S\left(\boldsymbol{\rho}_{A B} \mid\left\{B_{k}\right\}\right)\right],
$$

where $\left\{B_{k}\right\}$ denotes a complete set of projective measurements performed locally on subsystem $B$, and $S\left(\boldsymbol{\rho}_{A B} \mid\left\{B_{k}\right\}\right)=\sum_{k} p_{k} S\left(\boldsymbol{\rho}_{k}\right)$ is the quantum conditional entropy with $p_{k}=\operatorname{tr}_{A B}\left[\left(I \otimes B_{k}\right) \boldsymbol{\rho}_{A B}\left(I \otimes B_{k}\right)\right]$ and $\boldsymbol{\rho}_{k}=\operatorname{tr}_{B}\left[\left(I \otimes B_{k}\right) \boldsymbol{\rho}_{A B}\left(I \otimes B_{k}\right)\right] / p_{k}$. By substituting from Equations (13) and (15) into Equation (14), one can obtain the quantum discord as

$$
Q D\left(\boldsymbol{\rho}_{A B}\right)=S\left(\boldsymbol{\rho}_{B}\right)-S\left(\boldsymbol{\rho}_{A B}\right)+\min _{\left\{B_{k}\right\}}\left[\sum_{k} p_{k} S\left(\boldsymbol{\rho}_{k}\right)\right] .
$$

For a class of two-qubit states of qubits $A$ and $B$, i.e. the two-qubit $X$ states in standard basis $\{|11\rangle,|10\rangle,|01\rangle,|00\rangle\}$,

It is easy to show that

$$
\boldsymbol{\rho}_{A B}=\left(\begin{array}{cccc}
\rho_{11} & 0 & 0 & \rho_{14} \\
0 & \rho_{22} & \rho_{23} & 0 \\
0 & \rho_{32} & \rho_{33} & 0 \\
\rho_{41} & 0 & 0 & \rho_{44}
\end{array}\right) .
$$

$$
S\left(\boldsymbol{\rho}_{A B}\right)=-\sum_{i=1}^{4} \lambda_{i}, \quad S\left(\boldsymbol{\rho}_{B}\right)=\Gamma\left(\rho_{11}+\rho_{33}\right),
$$

where, $\lambda_{i}$ being the four eigenvalues of $\boldsymbol{\rho}_{A B}$ and $\Gamma(x)=-x \log _{2} x-(1-x) \log _{2}(1-x)$ is the Shannon entropy. According to Ref. [14] [27], $\min _{\left\{B_{k}\right\}}\left[\sum_{k} p_{k} S\left(\boldsymbol{\rho}_{k}\right)\right]$ can be obtained as

$$
\min _{\left\{B_{k}\right\}}\left[\sum_{k} p_{k} S\left(\boldsymbol{\rho}_{k}\right)\right]=\min \left[D_{1}, D_{2}\right],
$$

where

$$
D_{1}=\left(\rho_{11}+\rho_{33}\right) \Gamma\left(\frac{\rho_{11}}{\rho_{11}+\rho_{33}}\right)+\left(\rho_{22}+\rho_{44}\right) \Gamma\left(\frac{\rho_{22}}{\rho_{22}+\rho_{44}}\right),
$$




$$
D_{2}=\Gamma\left(\frac{1+\sqrt{\left(\rho_{11}+\rho_{22}-\rho_{33}-\rho_{44}\right)^{2}+4\left(\left|\rho_{14}\right|+\left|\rho_{23}\right|\right)^{2}}}{2}\right) .
$$

For our system under consideration above, for the density matrix in Equation (9), one can obtain the explicit expressions of the concurrence $(C)$ and quantum discord $(Q D)$ in the form

$$
\begin{aligned}
& C=2 \max \left[\left(\left|\rho_{23}\right|-\sqrt{\rho_{11} \rho_{44}}\right), 0\right], \\
& Q D=\min \left[Q D_{1}, Q D_{2}\right],
\end{aligned}
$$

where

$$
\begin{gathered}
Q D_{1}=\sum_{i=1}^{4} \lambda_{i} \log _{2} \lambda_{i}-\sum_{i} \rho_{i i} \log _{2} \rho_{i i} \\
Q D_{2}=\Gamma\left(\rho_{11}+\rho_{22}\right)+\sum_{i=1}^{4} \lambda_{i} \log _{2} \lambda_{i}+\Gamma\left(\frac{1+\sqrt{\left(\rho_{11}+\rho_{22}-\rho_{33}-\rho_{44}\right)^{2}+4\left|\rho_{23}\right|^{2}}}{2}\right),
\end{gathered}
$$

with

$$
\begin{aligned}
& \lambda_{1}=\rho_{11}, \\
& \lambda_{2}=\frac{1}{2}\left[\left(\rho_{22}+\rho_{33}\right)+\sqrt{\left(\rho_{22}-\rho_{33}\right)^{2}+4\left|\rho_{23}\right|^{2}}\right], \\
& \lambda_{3}=\frac{1}{2}\left[\left(\rho_{22}+\rho_{33}\right)-\sqrt{\left(\rho_{22}-\rho_{33}\right)^{2}+4\left|\rho_{23}\right|^{2}}\right], \\
& \lambda_{44}=\rho_{44},
\end{aligned}
$$

where, the non zero elements $\rho_{i j}$ are given in Equation (10).

Having obtained the results above, we are therefore in a position to discuss the dynamics behavior of the quantum discord and entanglement to the system consider in the following section.

\section{Numerical Results and Discussion}

Our goal in this article is to clarify what peculiar dynamics happened for quantum discord and entanglement (measured by concurrence) of the system under consideration as follow. In Figure 1, we plotted the concurrence $C$ as a function of scaled time $g t$ and $r$. It is apparent that when $0 \leq r \leq 0.33$ the concurrence is zero,

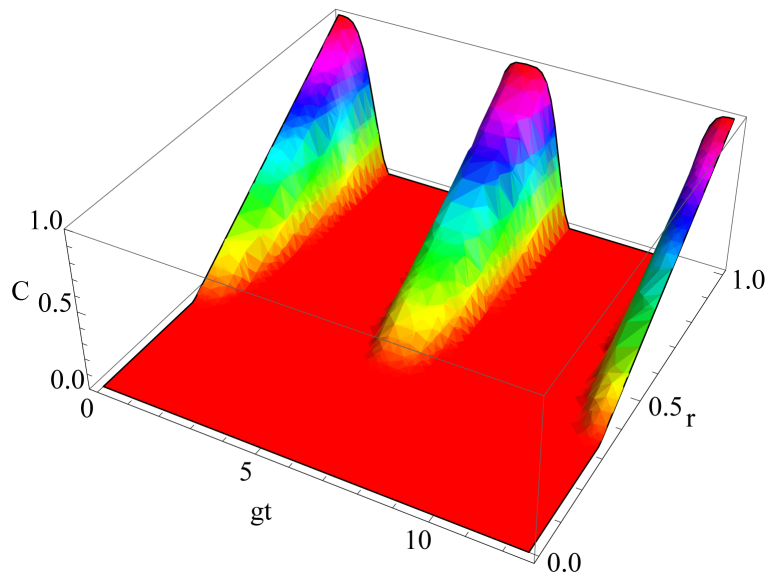

(a)

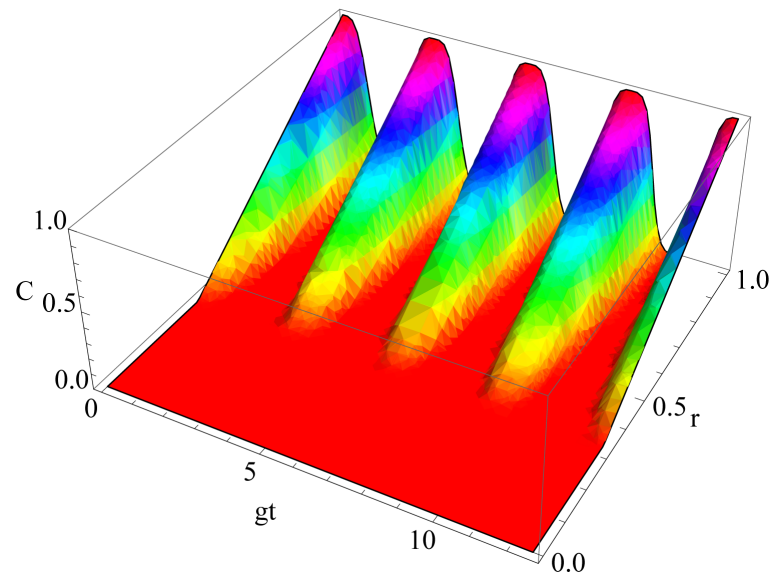

(b)

Figure 1. Concurrence $C$ plotted as a function of scaled time $g t$ and $r$, for (a) $p=1$ and (b) $p=2$. 
while $0.33<r \leq 1$, one can see that the concurrence evolves periodically and entanglement sudden death phenomenon occurs, as $r$ increases the local maximum value of the concurrence increases and tends to 1 when $r=1$. To study the effect of the atomic motion related to the field-mode structure parameter $p$ in entanglement, we compare between Figure 1(a) $(p=1)$ and Figure 1(b) $(p=2)$, it is observed that when $p$ increases the evolution period of the concurrence decreases and the time interval for zero entanglement becomes more and its wide becomes shorted. On the other hand, the quantum discord $(Q D)$ is plotted versus scaled time $g t$ and $r$ in Figure 2. The time behavior of quantum discord is periodical with period $2 \pi / p$, and its evolution periods are shorten with the increased parameter $p$. In order to compare between the quantum discord and concurrence, we plotted quantum discord and concurrence as a function of scaled time $g t$ for different values of $p$ and $r$ in Figure 3. We have two main regions responding $r$ : first region $0 \leq r \leq 0.33$, in this region the concurrence

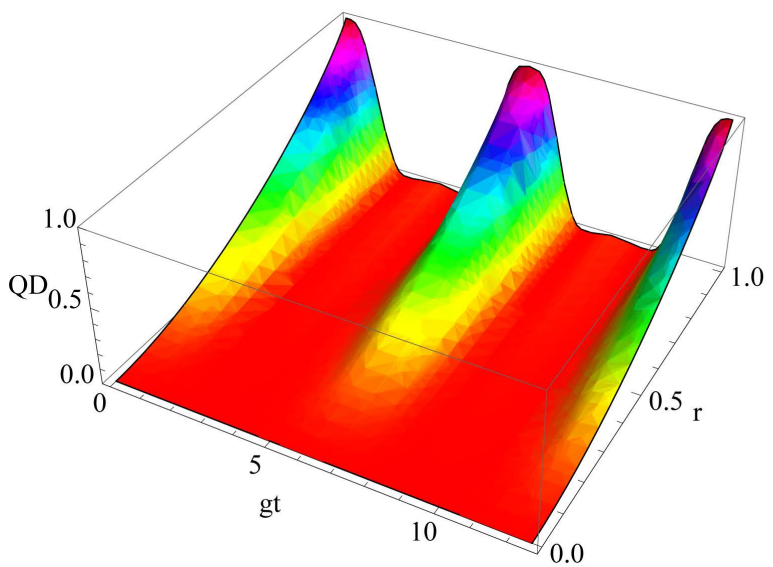

(a)

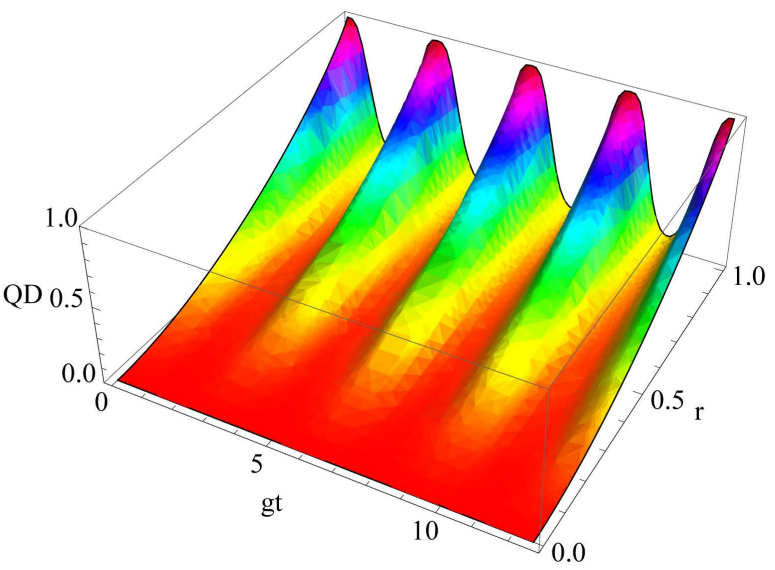

(b)

Figure 2. Quantum discord $Q D$ plotted as a function of scaled time $g t$ and $r$, for (a) $p=1$ and (b) $p=2$.

(a): $\mathrm{r}=0.3$ and $\mathrm{p}=1$

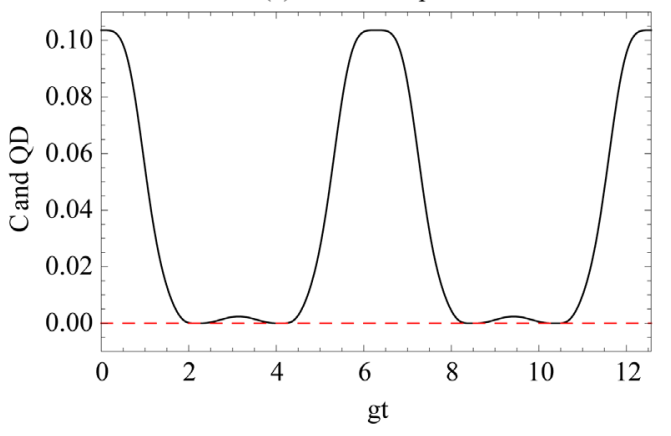

(c): $r=0.6$ and $p=1$

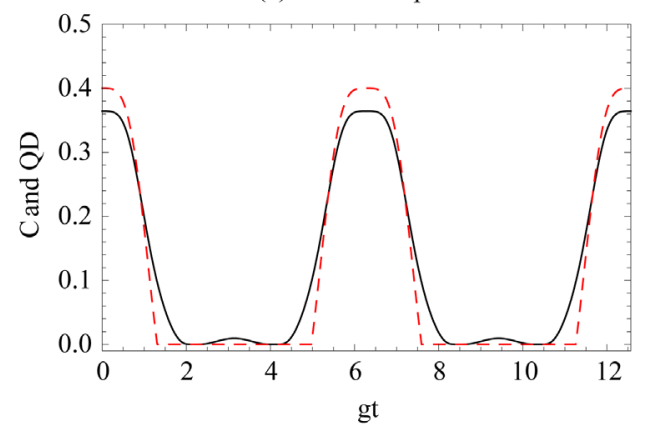

(b): $\mathrm{r}=0.3$ and $\mathrm{p}=2$

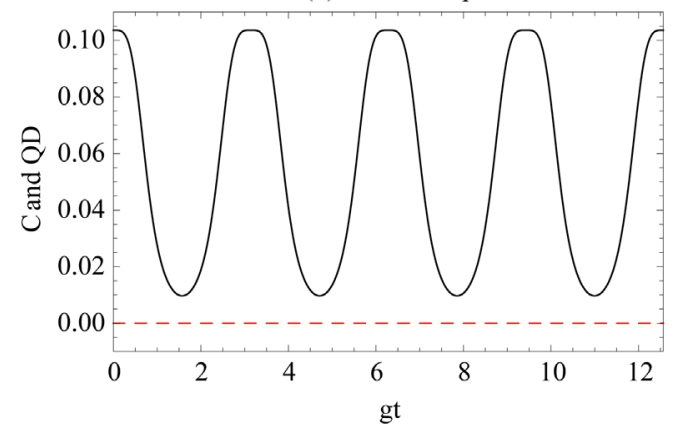

(d): $r=0.6$ and $p=2$

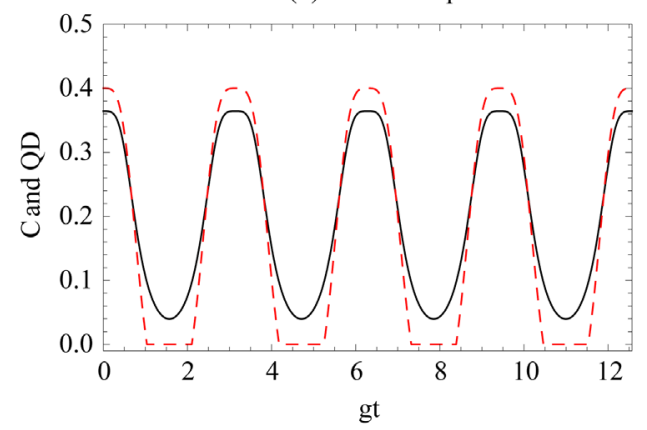

Figure 3. Concurrence $C$ and quantum discord $Q D$ versus scaled time $g t$. The black solid and red dashed lines denote respectively $Q D$ and $C$. 
always zero but the quantum discord remains non zero. Second region $0.33<r \leq 1$, the concurrence vanishes periodically and entanglement sudden death happens, while the quantum discord remains non zero with its corresponding fluctuations. Also the frequencies of quantifiers in unit time are the same for concurrence and quantum discord. Whereas the amplitude of concurrence is greater than the amplitude of quantum discord. However, the dynamic of quantum discord is quite different, although entanglement suddenly disappears during the evaluation, the quantum discord remains non zero. It means that the absence of entanglement does not indicate the absence of quantum discord, so quantum discord still can capture the quantum correlation between the two atoms when the entanglement is zero.

\section{Conclusion}

We have investigated the quantum discord and entanglement between two isolated two-level atoms which are initially prepared in Werner state and each atom interacts with a thermal cavity field by considering the atomic motion and the field-mode structure. It is shown that the entanglement vanishes periodically and sudden death of entanglement appears, while the quantum discord remains non zero with its corresponding fluctuations. We observe that the atomic motion and the field-mode structure have the same effect on the behavior of quantum discord and entanglement. Although entanglement suddenly disappears during the evaluation, the quantum discord remains non zero. Quantum discord still can capture the quantum correlation between the two atoms when the entanglement is zero.

\section{References}

[1] Bennett, C.H. and Di Vincenzo, D.P. (2000) Quantum Information and Computation. Nature, 404, 247. http://dx.doi.org/10.1038/35005001

[2] Mayer, D.A. (2000) Sophisticated Quantum Search without Entanglement. Physical Review Letters, 85, 4 p.

[3] Lanyon, B.P., Barbieri, M., Almeida, M.P. and White, A.G. (2008) Experimental Quantum Computing without Entanglement. Physical Review Letters, 101, Article ID: 200501. http://dx.doi.org/10.1103/PhysRevLett.101.200501

[4] Vedral, V. (2003) Classical Correlations and Entanglement in Quantum Measurements. Physical Review Letters, 90, Article ID: 050401. http://dx.doi.org/10.1103/PhysRevLett.90.050401

[5] Datta, A., Flammia, S.T. and Caves, C.M. (2005) Entanglement and the Power of One Qubit. Physical Review A, 72, Article ID: 042316. http://dx.doi.org/10.1103/PhysRevA.72.042316

[6] Ollivier, H. and Zurek, W.H. (2001) Quantum Discord: A Measure of the Quantumness of Correlations. Physical Review Letters, 88, Article ID: 017901. http://dx.doi.org/10.1103/PhysRevLett.88.017901

[7] Datta, A., Shaji, A. and Caves, C.M. (2008) Quantum Discord and the Power of One Qubit. Physical Review Letters, 100, Article ID: 050502. http://dx.doi.org/10.1103/PhysRevLett.100.050502

[8] Zidan, N. (2014) Quantum Discord of a Two-Qubit Anisotropy XXZ Heisenberg Chain with Dzyaloshinskii-Moriya Interaction. Journal of Quantum Information Science, 4, 104. http://dx.doi.org/10.4236/jqis.2014.42011

[9] Berrada, K., Fanchini, F.F. and Abdel-Khalek, S. (2012) Quantum Correlation between Each Qubit in a Two-Atom System and the Environment in Terms of Interatomic Distance. Physical Review A, 85, Article ID: 052315. http://dx.doi.org/10.1103/PhysRevA.85.052315

[10] Wang, C.-Z., Li, C.-X., Nie, L.-Y. and Li, J.-F. (2011) Classical Correlation and Quantum Discord Mediated by Cavity in Two Coupled Qubit. Journal of Physics B: Atomic, Molecular and Optical Physics, 44, Article ID: 015503. http://dx.doi.org/10.1088/0953-4075/44/1/015503

[11] Zhang, J.S. and Chen, A.X. (2012) Review of Quantum Discord in Bipartite and Multipartite Systems. Quantum Physics Letters, 1, 69.

[12] Mohamed, A.B. (2013) Pairwise Correlations via Quantum Discord and Its Geometric Measure in a Four-Qubit Spin Chain. Journal of the Egyptian Mathematical Society, 21, 68-74. http://dx.doi.org/10.1016/j.joems.2012.10.005

[13] Luo, S. (2008) Quantum Discord for Two-Qubit Systems. Physical Review A, 77, Article ID: 042303. http://dx.doi.org/10.1103/PhysRevA.77.042303

[14] Ali, M., Rau, A.R.P. and Alber, G. (2010) Quantum Discord for Two-Qubit X States. Physical Review A, 81, Article ID: 042105. http://dx.doi.org/10.1103/PhysRevA.81.042105

[15] Chen, Y.X., Li, S.W. and Yin, Z. (2010) Quantum Correlations in a Clusterlike System. Physical Review A, 82, Article ID: 052320. http://dx.doi.org/10.1103/PhysRevA.82.052320

[16] Hassan, A., Lari, B. and Joag, P. (2010) Thermal Quantum and Classical Correlations in a Two-Qubit XX Model in a 
Nonuniform External Magnetic Field. Journal of Physics A: Mathematical and Theoretical, 43, Article ID: 485302.

[17] Yuan, J.B., Kuang, L.M. and Liao, J.Q. (2010) Amplification of Quantum Discord between Two Uncoupled Qubits in a Common Environment by Phase Decoherence. Journal of Physics B: Atomic, Molecular and Optical Physics, 43, Article ID: 165503.

[18] Hao, X., Ma, C.L. and Sha, J. (2010) Decoherence of Quantum Discord in an Asymmetric-Anisotropy Spin System. Journal of Physics A: Mathematical and Theoretical, 43, Article ID: 425302.

[19] Sun, Z., Lu, X.M. and Song, L. (2010) Quantum Discord Induced by a Spin Chain with Quantum Phase Transition. Journal of Physics B: Atomic, Molecular and Optical Physics, 43, Article ID: 215504.

[20] Mi, Y.J. (2012) Classical Correlation and Quantum Discord in a Two-Qubit System under Dissipation Environments. International Journal of Theoretical Physics, 51, 544-553. http://dx.doi.org/10.1007/s10773-011-0933-5

[21] Ji, Y.H. and Liu, Y.M. (2013) Investigations into Quantum Correlation of Coupled Qubits in a Squeezed Vacuum Reservoir. Chinese Physics B, 22, Article ID: 020305.

[22] Galve, F., Giorgi, G.L. and Zambrini, R. (2011) Maximally Discordant Mixed States of Two-Qubits. Physical Review A, 83, Article ID: 012102. http://dx.doi.org/10.1103/PhysRevA.83.012102

[23] Girolami, D. and Adesso, G. (2011) Quantum Discord for General Two-Qubit States: Analytical Progress. Physical Review A, 83, Article ID: 052108. http://dx.doi.org/10.1103/PhysRevA.83.052108

[24] Schlicher, R.R. (1989) Jaynes-Cummings Model with Atomic Motion. Optics Communications, 70, 97-102. http://dx.doi.org/10.1016/0030-4018(89)90276-9

[25] Wootters, W.K. (1998) Entanglement of Formation of an Arbitrary State of Two-Qubits. Physical Review Letters, 80, 2245. http://dx.doi.org/10.1103/PhysRevLett.80.2245

[26] Henderson, L. and Vedral, V. (2001) Classical, Quantum and Total Correlations. Journal of Physics A: Mathematical and General, 34, 6899.

[27] Sarandy, M.S. (2009) Classical Correlation and Quantum Discord in Critical Systems. Physical Review A, 80, Article ID: 022108. http://dx.doi.org/10.1103/PhysRevA.80.022108 
Scientific Research Publishing (SCIRP) is one of the largest Open Access journal publishers. It is currently publishing more than 200 open access, online, peer-reviewed journals covering a wide range of academic disciplines. SCIRP serves the worldwide academic communities and contributes to the progress and application of science with its publication.

Other selected journals from SCIRP are listed as below. Submit your manuscript to us via either submit@scirp.org or Online Submission Portal.
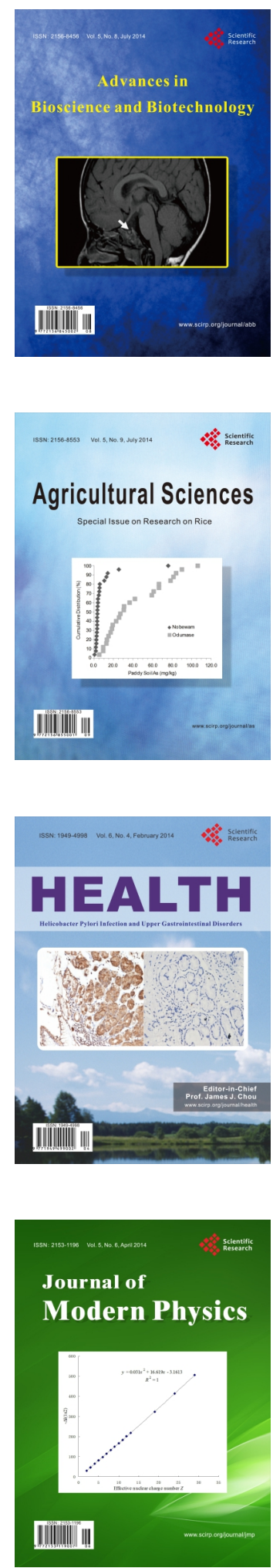
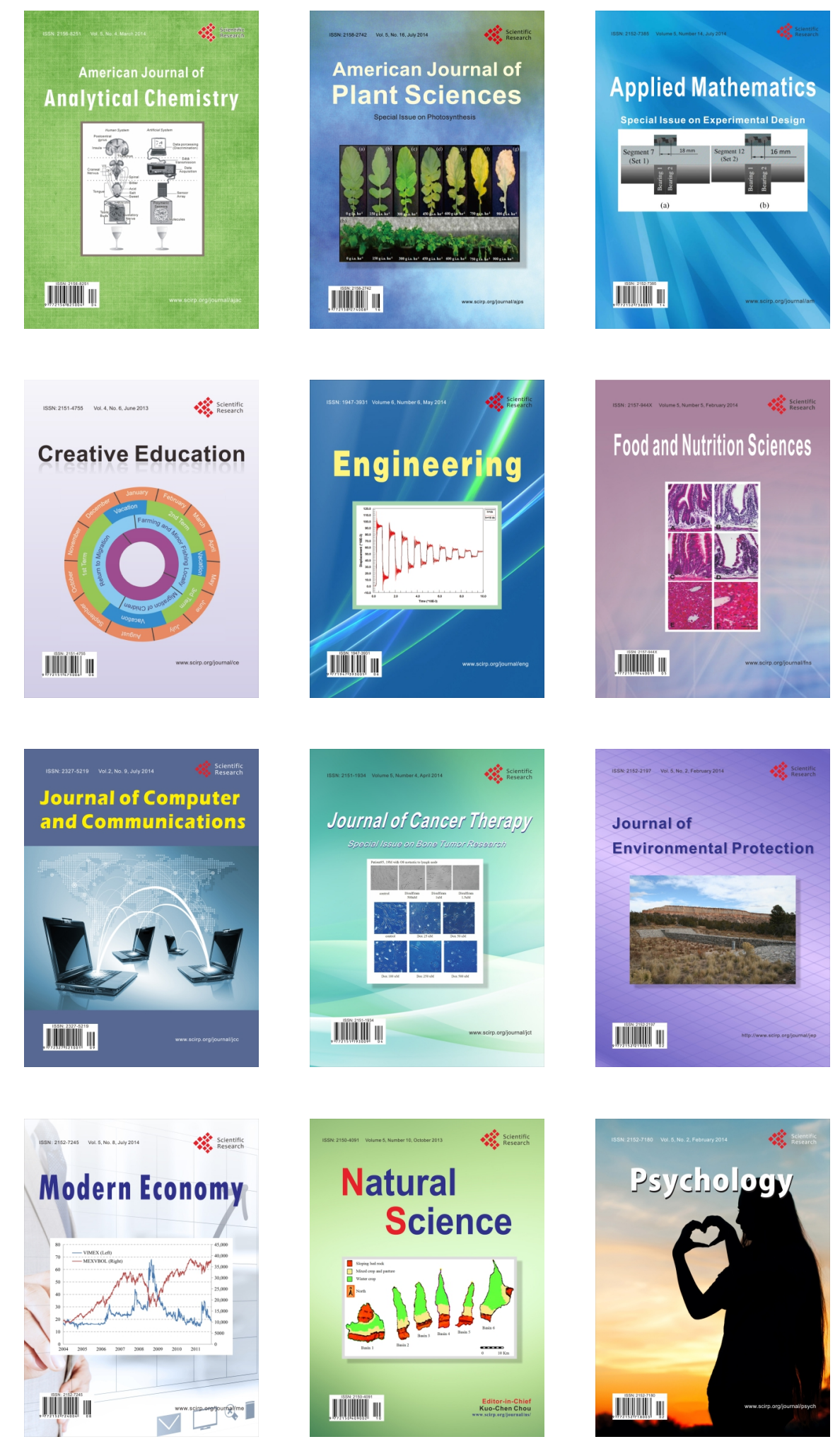